\title{
STRUCTURAL ARREST AND THERMODYNAMIC SCALING IN FILLER-REINFORCED POLYMERS
}

\author{
C. G. ROBERTSON \\ Bridgestone Americas, Center for Research and Technology, 1200 Firestone Parkway, Akron, OH 44317-0001 \\ R. Bogoslovov, C. M. Roland* \\ Chemistry Division, Code 6120, Naval Research Laboratory, Washington, DC 20375-5342
}

\begin{abstract}
The role of small silica particles on the stiffness and glass transition dynamics of polyvinylacetate (PVAc) was examined for filler volume fraction $(\phi)$ from 0 to 0.28 . Whereas the influences of bound polymer and a strain-dependent filler network were clearly noted in the shear properties, the only effect of filler on the bulk modulus was the reduction in deformable polymer. The calorimetric glass transition of PVAc and its dependence on cooling rate were unaltered by the presence of the silica, in agreement with previous dielectric relaxation results. In contrast to the temperature dependence of the segmental dynamics, which was independent of $\phi$, the effect of volume on segmental relaxation was amplified by the addition of silica. This resulted in larger values for the thermodynamic scaling exponent $(\gamma)$, which also increased sharply at the filler concentration corresponding to the development of a percolated filler network.
\end{abstract}

\section{INTRODUCTION}

Small-particle fillers generally increase both the stiffness and strength of rubber, as well as improving its processability. ${ }^{1-3}$ Development of novel fillers such as carbon nanotubes, ${ }^{4,5}$ nanoclay, ${ }^{6,7}$ graphene ${ }^{8,9}$ silica, ${ }^{10,11}$ and diamond ${ }^{12}$ nanoparticles has led to increased interest in polymer reinforcement. The effect of fillers on the mechanical response is very strain dependent. At all strains, stress amplification in the vicinity of the inextensible particles increases the modulus: ${ }^{13,14}$ however, when the filler concentration is sufficiently high, a network structure forms, giving rise to a larger and strongly nonlinear modulus. ${ }^{15-18}$ The nonlinearity is due to the mechanical lability of the flocculated particle structure, the breakup of which is governed by the strain energy. ${ }^{19-21}$

Interaction of the polymer chains in the vicinity of the filler interface can be expected to influence the former's local segmental dynamics, and there are various reports of glassy behavior induced in rubbery material due to proximity of filler particles such as carbon black. ${ }^{22-25}$ On the other hand, a recent review ${ }^{26}$ found that often there is no measurable effect on $T_{g}$ and that at least some conclusions regarding glassy interfacial dynamics are spurious. A characteristic property of the local segmental dynamics we investigate herein is the density scaling of the local segmental relaxation times. Commonly the complex interactions among molecules or chain segments are approximated as a spherically symmetric, two-body potential. Moreover, for local properties is it sufficient to neglect the slowly varying attractive term, so that the potential is represented by a simple inverse-power-law (IPL) repulsive potential ${ }^{27,28}$

$$
U(r)=A r^{-3 \gamma}
$$

in which $r$ is the intersegment distance, while $A$ and $\gamma$ are material constants (independent of thermodynamic conditions). To the extent the intermolecular potential can be represented by an IPL over the range of $r$ relevant to the segmental dynamics, all thermodynamic properties can be

* Corresponding author. Ph: 202-767-1719; Fax: 202-767-0594; email: Roland@nrl.navy.mil 
expressed as a function of $r^{3 \gamma}$ or equivalently $V^{\gamma}$, where $V$ is the specific volume. ${ }^{28,29,30}$ It follows that the local segmental relaxation times of polymers will depend only on the product variable $T V^{\prime}$,

$$
\tau_{\alpha}=f\left(T V^{\prime}\right)
$$

as has been demonstrated for more than 50 liquids and polymers. ${ }^{31,32}$ Notwithstanding this interpretation of the scaling as arising from an IPL approximation, relaxation times for the global chain modes of polymers are also a function of $T V^{\prime}$ with the same value of the exponent. ${ }^{33,34}$ This is an interesting result since the chain modes certainly involve substantial values of $r$ at which an IPL can no longer be accurate.

In this paper we analyze data on neat and silica-reinforced polyvinylacetate (PVAc), making use of density scaling to obtain information not amenable to direct experimental measurements. PVAc is not a general purpose elastomer due to its high $T_{g}$; however, the latter makes it convenient for carrying out measurements both above and below $T_{g}$. The silica consisted of $\sim 100 \mathrm{~nm}$ spherical particles, surface-treated to provide stronger interaction with the polymer.

\section{EXPERIMENTAL DETAILS}

Polyvinylacetate (Sigma-Aldrich; $M_{w}=167 \mathrm{~kg} / \mathrm{mol} ; M_{w} / M_{n}=2.01$ ) was mixed with NanO-Sil amorphous colloidal silica (Energy Strategy Associates, Inc.; spherical particles with average diameter of $\sim 100 \mathrm{~nm}$; surface treated with tetrasulfidosilane) using a Brabender PlastiCorder mixer at $70{ }^{\circ} \mathrm{C}$. Silica was incorporated into the PVAc at loadings from 0 to $28 \%$ (by volume). The final silica concentrations in the compounds were verified by thermogravimetric analysis. Good dispersion of the silica was affirmed for all filler concentrations from the optical transparency of the samples and by transmission electron microscopy (TEM). Representative TEM images are shown in Figure 1. Modulated differential scanning calorimetry (MDSC) was carried out using a TA Instruments Q100, calibrated with a sapphire standard. Samples were thoroughly dried prior to MDSC measurements. For measurement of $T_{g}$, data were collected during cooling of the samples in the calorimeter from 140 to $-10{ }^{\circ} \mathrm{C}$ at $1 \mathrm{C} / \mathrm{min}$ using a 1 degree modulation with a $60 \mathrm{~s}$ period. The cooling rate dependence of fictive temperature was also assessed using MDSC. For these experiments samples were cooled from 140 to $-10{ }^{\circ} \mathrm{C}$ in the calorimeter at various logarithmically spaced rates over the range 0.1 to $20 \mathrm{C} / \mathrm{min}$, with the fictive temperature determined from measurements during subsequent heating at $2 \mathrm{C} / \mathrm{min}$ (modulation amplitude and period same as for the $T_{g}$ measurements). Dielectric relaxation measurements employed a Novocontrol Alpha analyzer in combination with a Delta Design model 9023 oven. Values of the viscosity were measured at $70^{\circ} \mathrm{C}$ in a Mooney viscometer using the small rotor. Dynamic shear measurements on a TA Instruments ARES rheometer with a cone and plate geometry ( $15 \mathrm{~mm}$ diameter, $0.1 \mathrm{rad}$ angle) were carried out at $70{ }^{\circ} \mathrm{C}$. Strain sweeps from 0.01 to $10 \%$ strain amplitude were applied in dynamic shear using a frequency of $1 \mathrm{rad} / \mathrm{s}$. A Gnomix instrument was employed for pressure-volume-temperature measurements. The method is based on the confining fluid technique, with the sample surrounded by mercury. A detailed description of the apparatus can be found elsewhere. ${ }^{32}$ Isobaric volume measurements were made during cooling at a rate of $0.5 \mathrm{deg} / \mathrm{min}$ isobarically at hydrostatic pressures from 10 to $160 \mathrm{MPa}$. Isothermal runs were also conducted at a rate of $4 \mathrm{MPa} / \mathrm{min}$ for determination of the bulk modulus over pressures ranges from 10 to $80 \mathrm{MPa}$. 

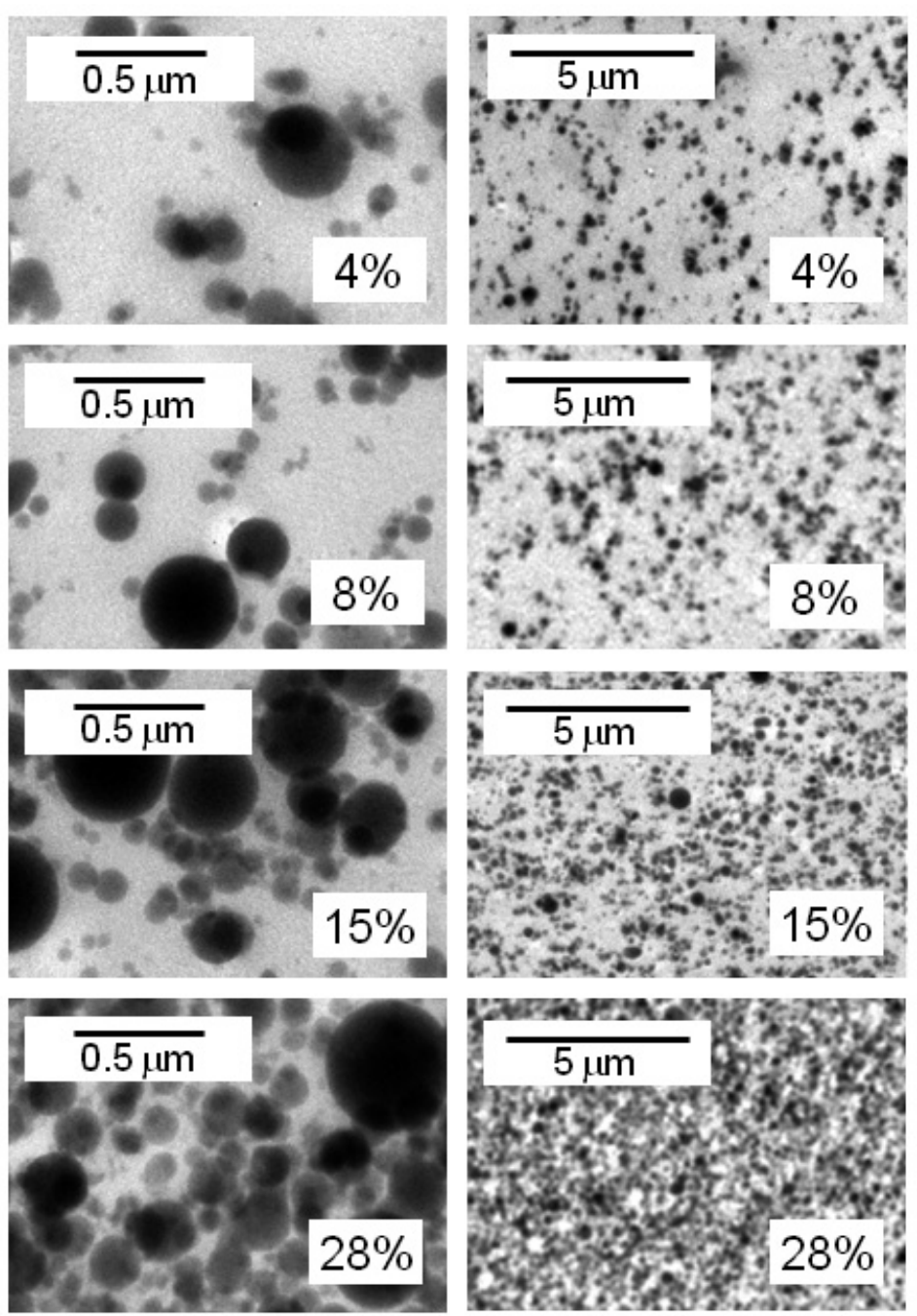

FIG. 1. - Transmission electron micrographs at two magnifications of PVAc with the indicated concentration of silica (vol.\%).

\section{RESULTS}

We studied the effect of silica particles on the viscosity and modulus of PVAc for filler concentrations from 0 to $28 \%$ by volume. A convenient metric of the filler-induced changes is to ratio the property of the filled polymer to its unfilled counterpart, and such reinforcement ratios are plotted in Figure 2 for the viscosity, shear modulus, and bulk modulus as a function of filler loading. Also plotted is the Guth-Gold expression, ${ }^{36}$ an extension of the Einstein viscosity equation with inclusion of a higher order term:

$$
\frac{\eta}{\eta_{0}}=\frac{G}{G_{0}}=1+2.5 \varphi+14.1 \phi^{2}
$$

In this equation $\phi$ is the volume fraction and the subscript zero denotes the unfilled polymer. 
Alternative expressions for the reinforcing effect of particles are reviewed elsewhere. ${ }^{37}$ For our purposes the Guth-Gold equation is used to estimate the hydrodynamic effect of the particles, and as seen in the figure, the experimental viscosity $(\eta)$ and dynamic shear storage modulus $\left(G^{\prime}\right)$ are larger than the prediction. This additional reinforcement can arise due to polymer chains bound to particles or occluded between aggregates, as well as from the formation of a continuous filler network. The latter only occurs at sufficient filler concentration, and this flocculated ("jammed") structure is highly sensitive to strain. These components of reinforcement are depicted schematically in Figure 3. The large strains in the Mooney viscosity measurement would break-up any filler network, so that we can ascribe viscosity enhancements above Equation 3 to bound and/or occluded polymer.

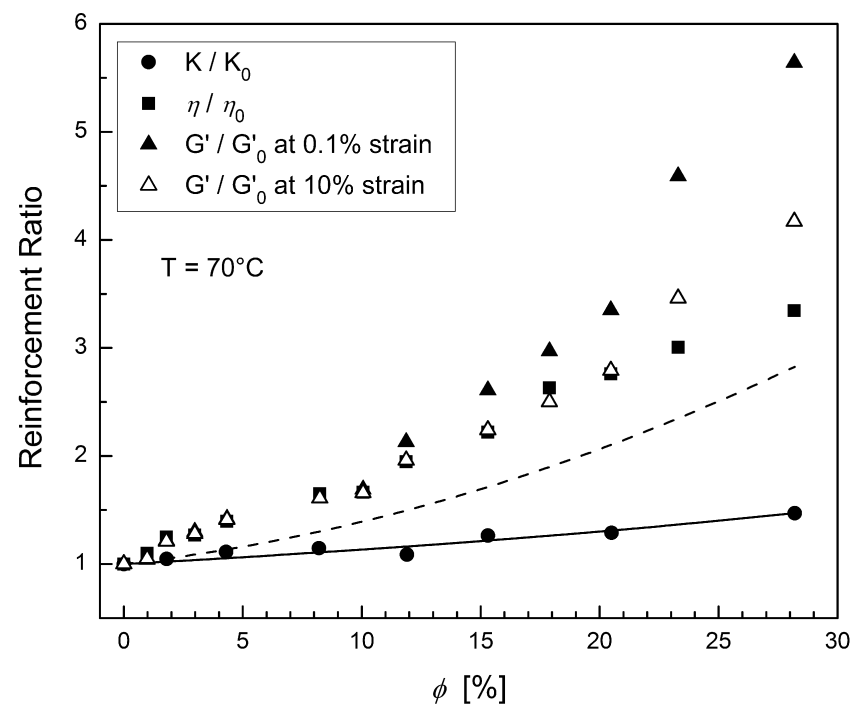

FIG. 2. - Comparison of the effect of filler on various properties. The dashed line is the Guth-Gold equation and the solid line is the bulk modulus calculated assuming only the polymer responds to pressure.

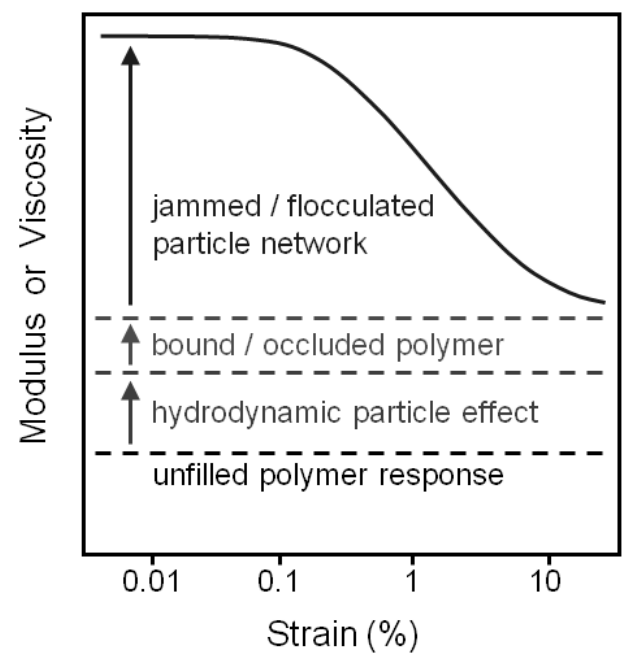

FIG. 3. - Illustration of reinforcement effects in particle-filled rubber. 
Even more pronounced is the modulus enhancement due to the filler network. The filler concentration dependence of the dynamic storage and loss moduli in the low strain region $(0.01$ to $0.1 \%$ dynamic amplitude) are plotted in Figure 4. Both quantities rise steeply around $\phi=11 \%$, denoting development of a flocculated network. The contribution of the latter is reflected in Figure 2 in greater departures from the Guth-Gold prediction. Break-up of the silica network, of course, is the cause of the difference between in $G^{\prime}$ for high and low strains at the higher filler concentrations in Figure 2.

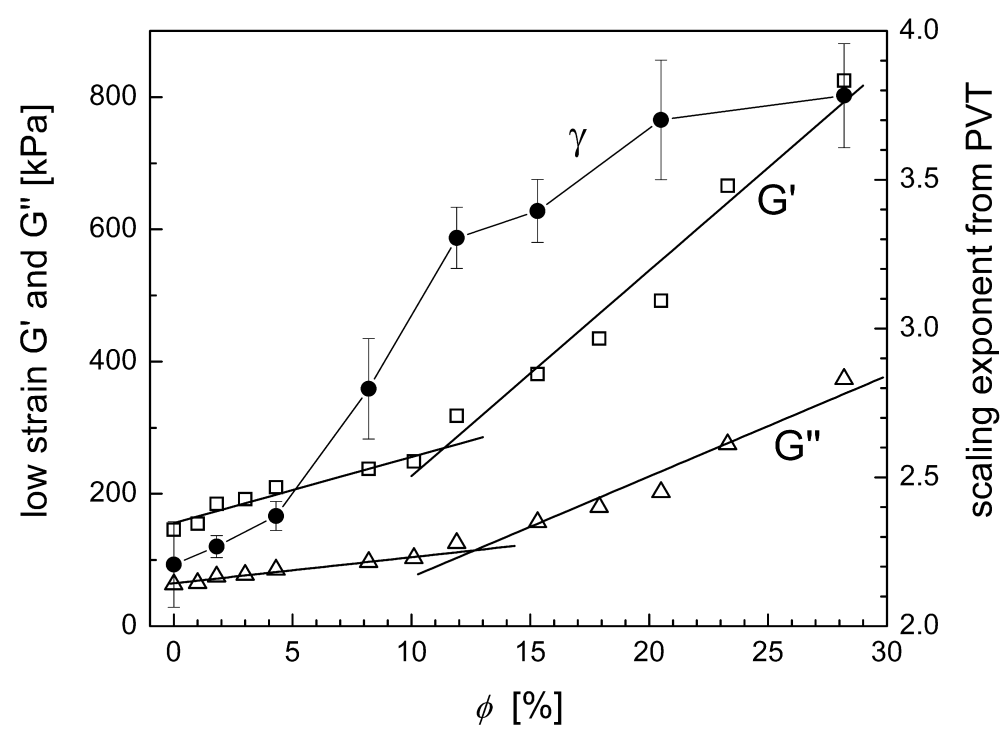

FIG. 4. - Change in dynamic moduli and scaling exponent with silica concentration.

There is a change in the dependence upon development of a filler network.

Unlike the substantial effects of filler on the shear viscosity and modulus, the $\phi$-dependence of the bulk modulus $(K)$ is quite weak (Figure 2), below even the hydrodynamic Guth-Gold values. The bulk modulus of the silica is approximately 30 times larger than $K$ of PVAc. Given this disparity, we assume that only the polymer is compressed under hydrostatic pressure, with the predicted $K$ seen (Figure 2) to be in good agreement with the experimental data. This result is consistent with other, unpublished results on a variety of filled elastomers, as well as data from Holownia ${ }^{38}$ on carbon black-filled polydienes. Generally the reinforcement mechanisms active in shear and uniaxial strain do not affect the bulk deformation behavior of filled polymers. The mechanisms depicted in Figure 3 are negligible for hydrostatic compression; the only effect of filler particles on $K$ is to reduce the concentration of deformable polymer.

In Figure 5 are shown the DSC results for the PVAc for various $\phi$. There is no change in the glass transition temperature due to the filler. Consistent with these results, the calorimetric fragility, defined as the change in the normalized fictive temperature with cooling rate ${ }^{39}$ is also invariant to silica content (Figure 6). Both results corroborate previous finding of no change in the local segmental relaxation times, measured by dielectric spectroscopy, in the presence of the filler (Figure 7). As shown in Figure 1, there is a significant amount of polymer-filler interface, especially at the higher filler loadings; nevertheless, the segmental relaxation dynamics for this interfacial material are not measurably different from that of neat PVAc. 


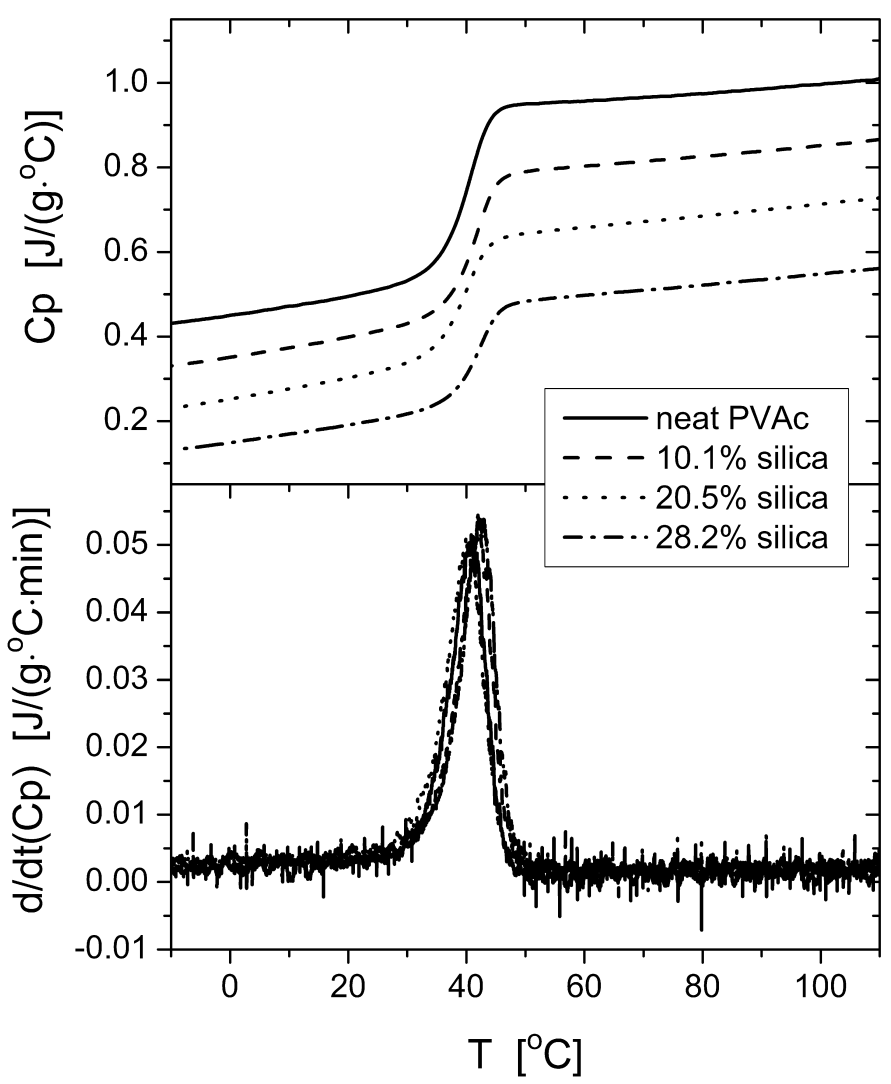

FIG. 5. - DSC results for neat and filled PVAc illustrating that $T_{g}$ is unaffected by the silica. The lower plot was normalized by the polymer content.

Dielectric spectroscopy is an attractive method to quantify the local segmental dynamics because the permittivity can be measured over 10 or more decades of frequency. However, for polymers containing carbon black or other conductive fillers, dielectric measurements are complicated by the contribution to the spectra from ionic conductivity. In such circumstance, the scaling exponent (Equation 2) can be determined from PVT measurements, without the need to superpose any experimental $\tau_{\alpha}$. This follows from the fact that the relaxation time at the glass transition is a constant, ${ }^{32}$ so that the scaling exponent is defined along the glass transition line, $T_{g}(P)$, according to

$$
\gamma=-\frac{V_{g}}{T_{g}} \frac{d T_{g}}{d V_{g}}
$$

Of course, the same $P V T$ data are necessary to determine $\gamma$ from superposition of $\tau_{\alpha}(T, V)$, so use of the equation simply obviates the need for measuring $\tau_{\alpha}$ at elevated pressures. Relaxation times measured at ambient pressure, in combination with the scaling relation, can be used to determine $\tau_{\alpha}$ for any pressure or for isochoric conditions. 


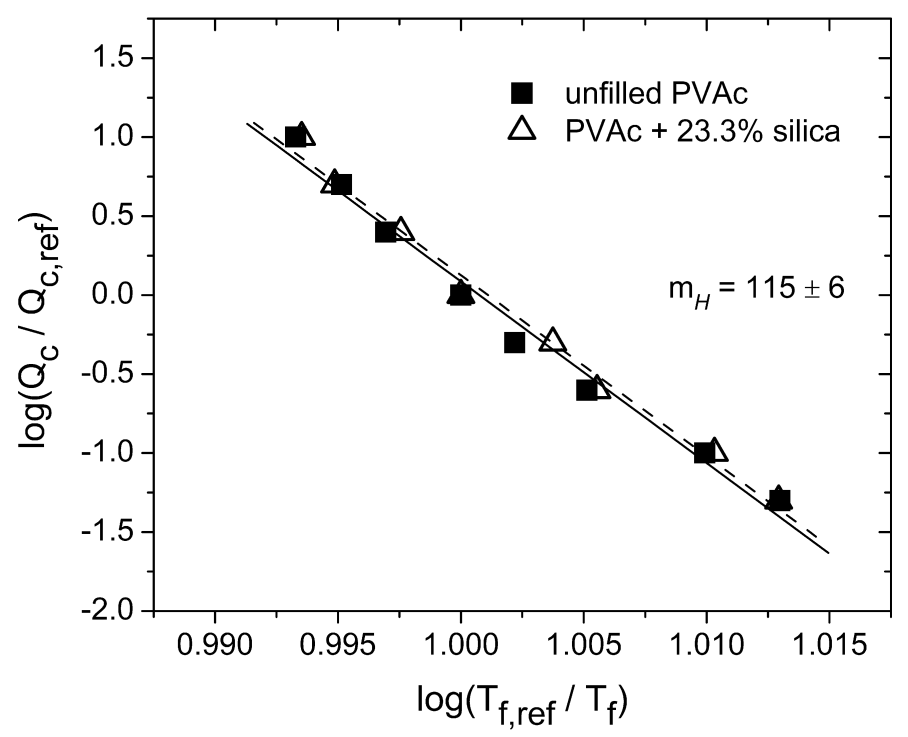

FIG. 6. - Cooling rate dependence of DSC fictive temperature for PVAc neat and with 23.3\% by volume silica. The latter has no effect on the calorimetric fragility.

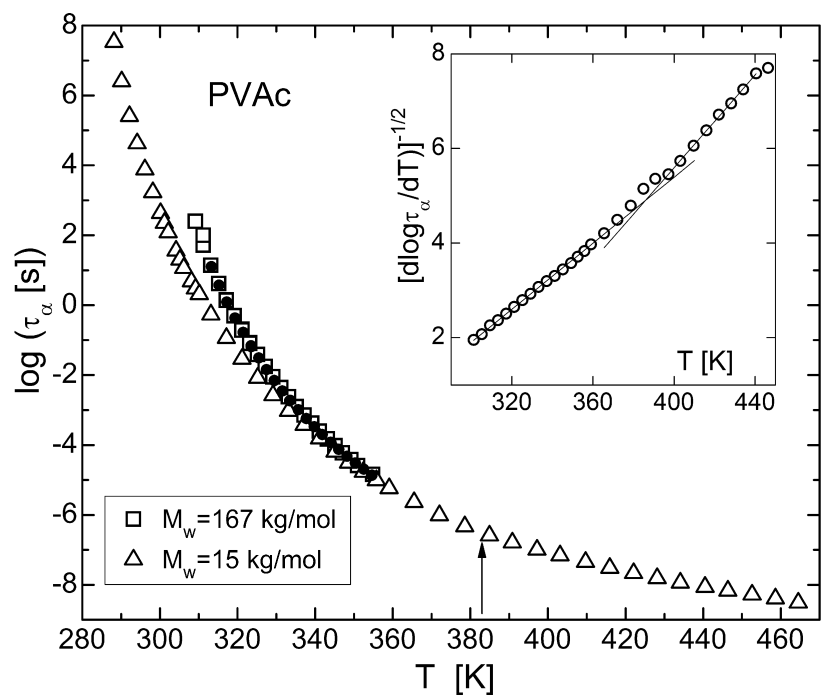

FIG. 7. - Temperature dependence of dielectric $\alpha$-relaxation times for the high $M_{w}$ unfilled PVAc (open squares) and a lower $M_{w}$ PVAc (open triangles) taken from Reference 40 . The difference between the two sets of data reflects the lower $T_{g}$ of the lower molecular weight sample. The latter are used because they traverse the dynamic crossover at $T=$ $T_{B}$ (indicated by the arrow). The inset is the Stickel plot showing the break at $T_{B} . \tau_{\alpha}$ are also shown for the high $M_{w}$ PVAc with $23 \%$ silica (solid circles); they are coincident with the data for the unfilled polymer.

The scaling exponents for the PVAc with varying silica content determined from PVT measurements are displayed in Figure 4. At the onset of an agglomerated filler network, there is increased sensitivity to volume, manifested as a change in the variation of $\gamma$ with $\phi$. (The specific volume used in Equation 4 was that of the polymer only. Using the total volume decreases $\gamma$ somewhat, making $\gamma$ a weaker function of $\phi$; however, the change in behavior at the percolation threshold remains.) Using the obtained values, $\gamma=2.21 \pm 0.14$ for the unfilled PVAc and $3.78 \pm$ 
0.17 for $\phi=28 \%$, the dependence of the relaxation times on the scaling variable $T V^{\gamma}$ are calculated (Figure 8). To extend the range of the dynamic data, we have made use of dielectric $\tau_{\alpha}$ reported by Richert ${ }^{40}$ for a lower molecular weight PVAc (see Figure 7).

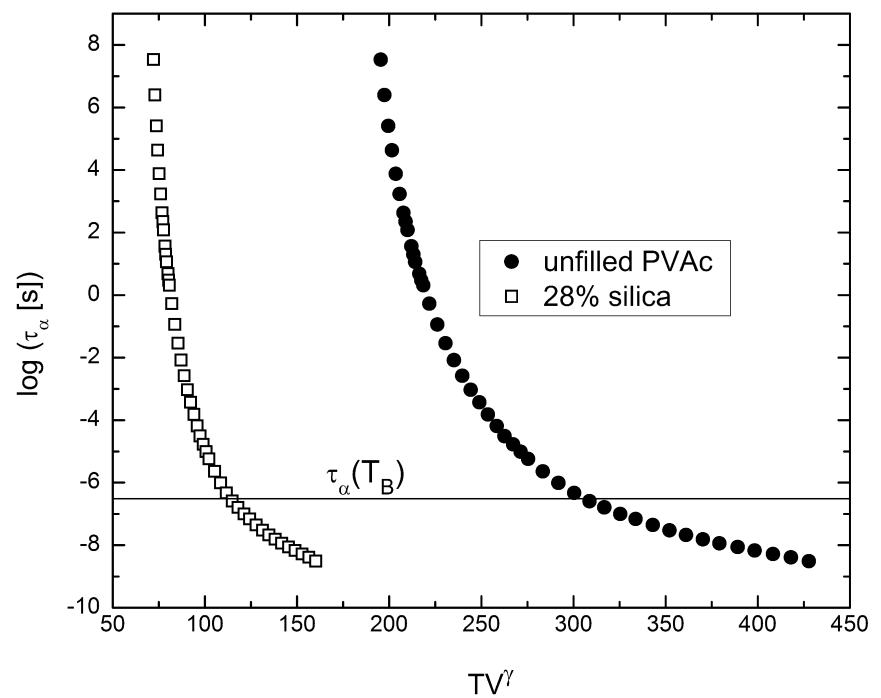

FIG. 8. - Scaled plot of segmental relaxation times using exponent determined from PVT measurements.

The horizontal line indicates the value at the dynamic crossover $\left(\log \left[\tau_{\alpha}\left(T_{B}\right) / \mathrm{s}\right]=-6.51\right)$.

The scaling relation enables the relaxation times to be deduced for any thermodynamic conditions, and in Figure 9 we use Equation 2 to calculate the variation of $\tau_{\alpha}$ with volume. Interestingly, although the relaxation times and their temperature dependence at low pressures are insensitive to filler (Figure 7), the volume-dependence is markedly affected by the silica.

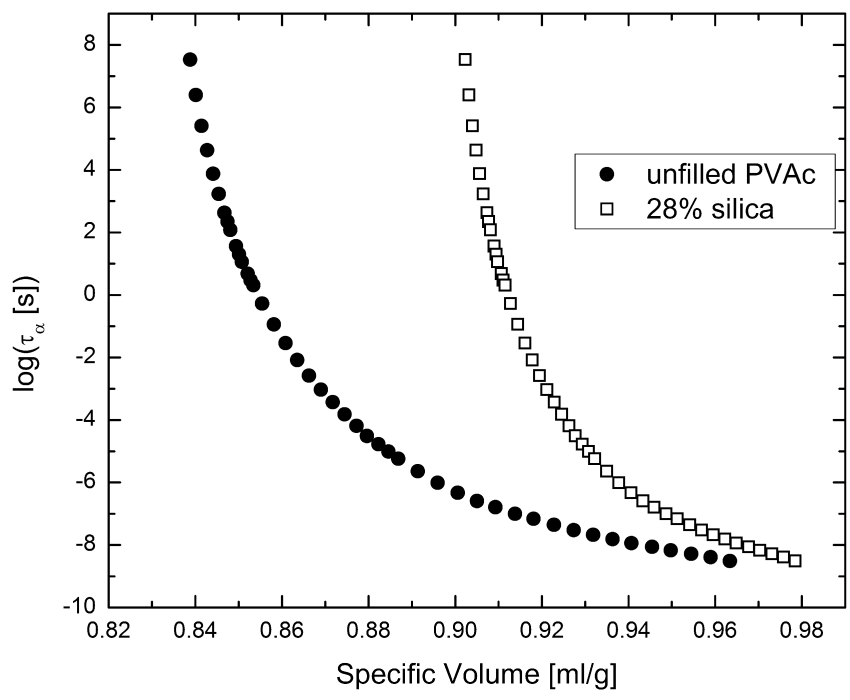

FIG. 9. — Volume-dependences for PVAc neat and with $\phi=0.28$.

An interesting phenomenon in both molecular liquids and polymers is the characteristic change in the dynamics at a temperature, $T_{B}$, above $T_{g}$. This "dynamic crossover" is manifested 
as a change in the temperature dependence of the dielectric strength (reflecting the amplitude of the molecular motion), which decreases much more rapidly with temperature above $T_{B} .{ }^{39}$ Other properties, including the breadth of the relaxation function and the relaxation times show similar changes in temperature dependence at $T_{B}{ }^{41}$ The origin of the dynamic crossover is still debated, with various models offering different interpretations: (i) a liquid-liquid transition as postulated for polymers by Boyer; ${ }^{42,43}$ (ii) a crossover from free diffusion to highly cooperative diffusion, as predicted by energy landscape models, first advanced by Goldstein; ${ }^{44}$ (iii) percolation of "liquid-like cells", according to the Cohen-Grest free-volume model; ${ }^{45,46}$ (iv) a marked increase in the degree of intermolecular cooperation, according to the coupling model of Ngai; ${ }^{47,48}$ and (vi) the divergence of the viscosity according to Mode Coupling Theory. ${ }^{49,50}$

The usual method of determining $T_{B}$ is from the model-independent derivative function of Stickel et al. ${ }^{51}$

$$
f_{\text {Stickel }}=\left[\frac{d \log (\tau)}{d(1000 / T)}\right]^{-\frac{1}{2}}
$$

which yields a straight line whose slope changes at $T_{B}$. For PVAc at ambient pressure, $T_{B}=383$ $\mathrm{K},{ }^{47}$ for which $\tau_{\alpha}=3.1( \pm 0.2) \times 10^{-7} \mathrm{~s}$. This value of the relaxation time is indicated in Figure 8. An interesting aspect of the dynamic crossover is that the relaxation time is constant at $T_{B}$; that is, while higher pressures increase the temperature of the crossover, $\tau_{\alpha}\left(T_{B}\right)$ remains the same. ${ }^{52,53}$ Using this fact, we can calculate the effect of filler on the pressure-dependence of $T_{B}$ for the PVAc. Since $\tau_{\alpha}$ is defined by the quantity $T V^{\prime}$, we calculate for various pressures the temperature at which $\tau_{\alpha}\left(T_{B}\right)=3.1 \times 10^{-7} \mathrm{~s}$. The results are shown in Figure 10. Reflecting the stronger influence of volume (larger value of the scaling exponent), the temperature of the dynamic crossover increases more rapidly with pressure in the presence of the silica filler.

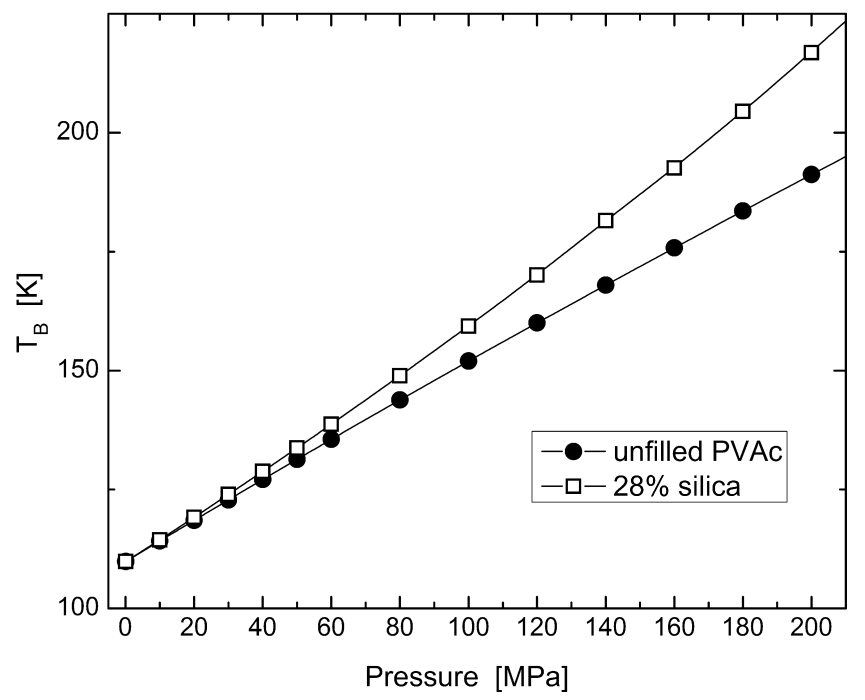

FIG. 10. - Temperature of the dynamic crossover for filled and unfilled PVAc (calculated assuming $\tau_{\alpha}$ at $T_{B}$ is independent of pressure). 


\title{
SUMMARY
}

In filled polymers, there are different variables to control the reinforcement, such as the size and concentration of the particles, and the nature of the polymer-filler interface. The presence of bound polymer and the introduction of a jammed filler network can enhance the modulus significantly beyond the hydrodynamic effect of particle inclusions, at least for shear and tensile modes of deformation. However, for hydrostatic pressure there is no relative displacement between polymer chains and the particles, and thus the same fillers do not "reinforce" the bulk modulus. The only effect on the resistance to pressure is due to the decrease in the amount of deformable polymer. It was suggested by Leaderman ${ }^{54}$ that intramolecular motions control the bulk modulus, while the shear modulus is governed by the intermolecular (intersegmental) dynamics. This is consistent with our earlier finding that the strength of the dielectric normal mode of polymers is insensitive to volume changes. ${ }^{55}$ The inference is that the chains accommodate hydrostatic pressure by a reduction in interchain distances, with minimal change in the molecular (chain coil) size per se. The idea is that the bulk modulus arises from van der Waals interactions, whereas rubber extension is largely an entropic process. ${ }^{56}$ (Although the two processes are coupled, since shear strains require moving other molecules aside, so that even far from where the molecular reorientation occurs, an added pressure is felt due to an elastic misfit.) This implies that calculations, for example of Poisson's ratio, based on interconversion between the modes should be approached with caution due to the dissimilarity of the mechanisms. Recent studies have highlighted these differences. ${ }^{57-59}$ Our results for filled PVAc emphasize the difference in the molecular origins for shear and hydrostatic deformation, evident in the very different effects of particle reinforcement.

Nearly 40 years ago, Kraus and Gruver ${ }^{60}$ stated: "It is well known that the glass transition of high polymers is virtually unaffected by incorporation of reinforcing fillers at technologically important loadings." Although some studies have concluded otherwise,${ }^{26}$ the present findings for silica-filled PVAc and our other recent work ${ }^{11,61}$ support this conclusion. However, the invariance of $T_{g}$, its rate dependence, and the low pressure segmental relaxation properties to filler notwithstanding, the segmental relaxation times become more sensitive to volume in the presence of filler. There is an abrupt increase in the scaling exponent, characterizing this volume-dependence, at a filler concentration associated with formation of a percolated filler network. One consequence is an increase in the pressure-dependence of the dynamic crossover temperature, demarcating the onset of strong intermolecular cooperation of the dynamics. These results may be connected to the observation that filler slows the rate of structural relaxation in the glassy state; ${ }^{62,63}$ such physical aging is governed by the loss of volume. Current efforts are directed to assessing whether the enhanced volume sensitivity of the segmental dynamics is a general phenomenon in filled polymers.

\section{ACKNOWLEDGEMENT}

The work at NRL was supported by the Office of Naval Research. CGR thanks Bridgestone Americas for permission to publish and RB the American Society of Engineering Education for a post-doctoral appointment. Enlightening comments of P.H. Mott are gratefully acknowledged. We thank P. Sadhukhan for TEM images.

\section{REFERENCES}

\author{
${ }^{1}$ A. I. Medalia, Rubber Chem. Technol. 60, 45 (1987). \\ ${ }^{2}$ W. H. Waddell and L.R. Evans, Rubber Chem. TeChnol. 69, 377 (1996). \\ ${ }^{3}$ G. Hamed, Rubber Chem. Technol. 73, 524 (2000); ibid 80, 533 (2007).
}


${ }^{4}$ L. Bokobza, Polymer 48, 4907 (2007).

${ }^{5}$ J. N. Coleman, U. Khan, W. J. Blau and Y. K Gunko, Carbon 44, 1624 (2006).

${ }^{6}$ Y. T. Vu, J. E. Mark, L. H. Pham, and M. Engelhardt, J. Appl. Polym. Sci. 82, 1391 (2001).

${ }^{7}$ S. Sadhu and A. K. Bhowmick, Rubber Chem. Technol. 76, 860 (2003).

${ }^{8}$ S. Stankovich, D. A. Dikin, G. H. B. Dommett, K. M. Kohlhaas, E. J. Zimney, E. A. Stach, R. D. Piner, S. T. Nguyen, and R. S. Ruoff, Nature 442, 282 (2006).

${ }^{9}$ K. Wakabayashi, C. Pierre, D. A. Dikin, R. S. Ruoff, T. Ramanathan, L. C. Brinson, and J. M. Torkelson, Macromolecules 41, 1905 (2008).

${ }^{10}$ A. Bansal, H. C. Yang, C. Z. Li, K. W. Cho, B. C. Benicewicz, S. K. Kumar, and L. S. Schadler, Nature Materials 4, 693 (2005).

${ }^{11}$ R. B. Bogoslovov; C. M. Roland; A. R. Ellis; A. M. Randall; C. G. Robertson, Macromolecules 41, 1289 (2008).

${ }^{12}$ K. D. Behler, A. Stravato, V. Mochalin, G. Korneva, G. Yushin, and Y. Gogotsi, ACS Nano 3, 363 (2009).

${ }^{13}$ G. Kraus, J. Poly. Sci.: Part B.: Polym. Lett. 8, 601 (1970).

${ }^{14}$ A. I. Medalia, Rubber Chem. Technol. 47, 411 (1974).

${ }^{15}$ A. R. Payne, J. Appl. Polym. Sci. 6, 57 (1962).

${ }^{16}$ W. P. Fletcher and A. N. Gent, Trans. Inst. Rubber Ind. 29, 266 (1953).

${ }^{17}$ S. D. Gehman, D. E. Woodford, and R. B. Stambaugh, Ind. Eng. Chem. 33,1032 (1941).

${ }^{18}$ C. M. Roland, J. Rheol. 34, 25 (1990).

${ }^{19}$ C. G. Robertson and X. Wang, Phys. Rev. Lett. 95, 075703 (2005).

${ }^{20}$ X. R. Wang and C. G. Robertson, Phys. Rev. E 72, 031406 (2005).

${ }^{21}$ C. G. Robertson, R. Bogoslovov, and C. M. Roland, Phys. Rev. E 75, 051403 (2007).

${ }^{22}$ H. Montes, F. Lequeux, and J. Berriot, Macromolecules 36, 8107 (2003).

${ }^{23}$ M.-J. Wang, Rubber CHEM. TeChnol. 71, 520 (1998).

${ }^{24}$ M. A. Kader, K. Kim, Y.-S. Lee, C. Nah, J. Matl. Sci. 41, 7341 (2006).

${ }^{25}$ S. Bandi and D. A. Schiraldi, Macromolecules 39, 6537 (2006).

${ }^{26}$ C. G. Robertson and C. M. Roland, Rubber Chem. Technol. 81, 506 (2008).

${ }^{27}$ H. C. Longuet-Higgins and B. Widom, Mol. Phys. 8, 549 (1964).

${ }^{28}$ W. G. Hoover and M. Ross, Contemp. Phys. 12, 339 (1971).

${ }^{29}$ D. Coslovich and C.M. Roland, J. Phys. Chem. B 112, 1329 (2008).

${ }^{30}$ D. Coslovich and C.M. Roland, J. Chem. Phys. 130, 014508 (2009).

${ }^{31}$ R. Casalini and C.M. Roland, Phys. Rev. E 69, 062501 (2004).

${ }^{32}$ C. M. Roland, S. Hensel-Bielowka, M. Paluch and R. Casalini, Rep. Prog. Phys. 68, 1405 (2005).

${ }^{33}$ C. M. Roland, R. Casalini, and M. Paluch, J. Polym. Sci. Polym. Phys. Ed. 42, 4313 (2004).

${ }^{34}$ R. Casalini and C. M. Roland, Macromolecules 38, 1779 (2005).

${ }^{35}$ P. Zoller and D. J. Walsh, Standard Pressure-Volume-Temperature Data for Polymers; Technomic, Lancaster, PA, 1995.

${ }^{36}$ E. Guth, J. Appl. Phys. 16, 20 (1945).

${ }^{37}$ D. J. Kohls and G. Beaucage, Curr. Opin. Solid State Mater. Sci. 6, 183 (2002).

${ }^{38}$ B. P. Holownia, Rubber Chem. Technol. 48, 246 (1975).

${ }^{39}$ C. G. Robertson, P. G. Santangelo, and C. M. Roland, J. Non-Cryst. Solids 275, 153 (2000).

${ }^{40}$ R. Richert, Physcia A 287, 26 (2000).

${ }^{41}$ C. A. Angell, K. L. Ngai, G. B. McKenna, P. F. McMillan, S. W. Martin, J. Appl. Phys. 88, 3113 (2000).

${ }^{42}$ R. F. Boyer, J. Polym. Sci. Polym. Phys. Ed. 23, 21 (1985).

${ }^{43}$ D. J. Plazek, J. Polym. Sci. Polym. Phys. Ed. 20, 1533 (1982); D.J. Plazek, G.F. Gu, ibid. 20, 1551 (1982); J. Chen, C.Kow, L.J. Fetters, D.J. Plazek, ibid.20, 1565 (1982); S.J. Orbon, D.J. Plazek, ibid. 20, 1575 (1982).

${ }^{44}$ M. Goldstein, J. Chem. Phys. 51, 4767 (1969). 
${ }^{45}$ M. H. Cohen, G. S. Grest, Phys. Rev. B 20, 1077 (1979); G. S. Grest, , M. H. Cohen, Adv. Chem. Phys. 48, 455 (1981). ${ }^{46}$ M. Paluch, R. Casalini and C. M. Roland, Phys. Rev. E 67, 021508 (2003).

${ }^{47}$ K. L. Ngai, C. M. Roland, Polymer 43, 567 (2002).

${ }^{48}$ R. Casalini, K. L. Ngai, C. M. Roland, Phys. Rev. B 68, 014201 (2003).

${ }^{49}$ W. Götze, L. Sjogren, Rep. Prog. Phys. 55, 241 (1992).

${ }^{50}$ W. Götze, J. Phys.: Cond. Matt. 11, A1 (1999).

${ }^{51}$ F. Stickel, E. W. Fischer, R. Richert, J. Chem. Phys. 102, 6251 (1995).

${ }^{52}$ R. Casalini, M. Paluch, C. M. Roland, J. Chem. Phys. 118, 5701 (2003).

${ }^{53}$ R. Casalini, C. M. Roland, Phys. Rev. Lett. 92, 245702 (2004).

${ }^{54}$ H. Leaderman," "Viscoelasticity Phenomena In Amorphous High Polymeric Systems" in Rheology:Theory and Applications, Vol. 2; F. R. Eirich, Ed.; Academic Press: New York, 1958. p. 1-61.

${ }^{55}$ R. Casalini and C.M. Roland, Macromolecules 38, 1779 (2005).

${ }^{56}$ D. Tabor, Polymer 35, 2759 (1994).

${ }^{57}$ G. A. Alvarez, M. W. Quintero, S. Eckert-Kastner, T. Alshuth, and H. Reinecke, J. Poly. Sci.: Part B: Polym. Phys. 45, 91 (2007).

${ }^{58}$ Y. Meng and S. L. Simon, J. Poly. Sci.: Part B: Polym. Phys. 45, 3375 (2007).

${ }^{59}$ P. H. Mott, J. R. Dorgan, and C. M. Roland, J. Sound Vib. 312, 572 (2008).

${ }^{60}$ G. Kraus and J. T. Gruver, J. Polym. Sci.: Part A-2: Polym. Phys. 8, 571 (1970).

${ }^{61}$ C. G. Robertson, C. J. Lin, M. Rackaitis, and C. M. Roland, Macromolecules 41, 2727 (2008).

${ }^{62}$ P. Rittigstein and J. M. Torkelson, J. Poly. Sci.: Part B: Polym. Phys. 44, 2935 (2006).

${ }^{63}$ S. Amanuel, A. N. Gaudette, and S. S. Sternstein, J. Poly. Sci.: Part B: Polym. Phys. 46, 2733 (2008). 